\title{
Deregulation of phytoene- $\beta$-carotene synthase results in derepression of astaxanthin synthesis at high glucose concentration in Phaffia rhodozyma astaxanthin-overproducing strain MK19
}

\author{
Lili Miao ${ }^{1 *} \mathbb{D}$, Shuang $\mathrm{Chi}^{2}$, Mengru Wu${ }^{1}$, Zhipei Liu ${ }^{1}$ and Ying Li
}

\begin{abstract}
Background: A major obstacle to industrial-scale astaxanthin production by the yeast Phaffia rhodozyma is the strong inhibitory effect of high glucose concentration on astaxanthin synthesis. We investigated, for the first time, the mechanism of the regulatory effect of high glucose $(>100 \mathrm{~g} / \mathrm{L})$ at the metabolite and transcription levels.

Results: Total carotenoid, $\beta$-carotene, and astaxanthin contents were greatly reduced in wild-type JCM9042 at high $(110 \mathrm{~g} / \mathrm{L})$ glucose; in particular, $\beta$-carotene content at $24-72 \mathrm{~h}$ was only $14-17 \%$ of that at low $(40 \mathrm{~g} / \mathrm{L})$ glucose. The inhibitory effect of high glucose on astaxanthin synthesis appeared to be due mainly to repression of lycopene-to$\beta$-carotene and $\beta$-carotene-to-astaxanthin steps in the pathway. Expression of carotenogenic genes crtE, pbs, and ast was also strongly inhibited by high glucose; such inhibition was mediated by creA, a global negative regulator of carotenogenic genes which is strongly induced by glucose. In contrast, astaxanthin-overproducing, glucose metabolic derepression mutant strain MK19 displayed de-inhibition of astaxanthin synthesis at $110 \mathrm{~g} / \mathrm{L}$ glucose; this de-inhibition was due mainly to deregulation of pbs and ast expression, which in turn resulted from low creA expression. Failure of glucose to induce the genes reg1 and $h x k 2$, which maintain CreA activity, also accounts for the fact that astaxanthin synthesis in MK19 was not repressed at high glucose.

Conclusion: We conclude that astaxanthin synthesis in MK19 at high glucose is enhanced primarily through derepression of carotenogenic genes (particularly pbs), and that this process is mediated by CreA, Reg1, and Hxk2 in the glucose signaling pathway.
\end{abstract}

Keywords: Phaffia rhodozyma, Glucose metabolism, Astaxanthin, Phytoene- $\beta$-carotene synthase, Gene expression

\section{Background}

Astaxanthin is an orange-red carotenoid pigment that has great commercial value because of its antioxidant and coloration properties. It is utilized increasingly as a protective agent against in vivo oxidative damage [1-5], and as a colorant for aquatic food products such as salmon, lobster, and crab. It can eliminate free radicals with antioxidant activity $\sim 500$-fold higher than that of

\footnotetext{
* Correspondence: miaoll@im.ac.cn

${ }^{1}$ State Key Laboratory of Microbial Resources, Institute of Microbiology, Chinese Academy of Sciences, No. 1 West Beichen Road, Chaoyang District, Beijing 100101, People's Republic of China

Full list of author information is available at the end of the article
}

vitamin E [6-10]. It has also been reported to inhibit cell toxicity mediated by reactive oxygen species (ROS), to support immune system processes, and to exert antiaging and anti-cancer effects. For these reasons, there is increasing demand for astaxanthin, particularly from natural sources.

The yeast Phaffia rhodozyma (sexual form, Xanthophyllomyces dendrorhous; also referred to hereafter as $P$. rhodozyma to avoid confusion) is one of the best natural sources of astaxanthin, because of its capacity for fermentation of glucose via rapid heterotrophic metabolism, and for high cell densities in autofermentors [11-

(C) The Author(s). 2019 Open Access This article is distributed under the terms of the Creative Commons Attribution 4.0 International License (http://creativecommons.org/licenses/by/4.0/), which permits unrestricted use, distribution, and 
13]. P. rhodozyma is a strong fermenter of sugars (e.g., glucose, sucrose, raffinose) -- a desirable property in regard to industrial production. On the other hand, it displays strong Crabtree effect (production of large amounts of fermentation byproducts such as ethanol and acetate, resulting in low biomass), and greatly reduced astaxanthin synthesis at glucose concentrations > $40 \mathrm{~g} / \mathrm{L}[14,15]$.

Biosynthesis of carotenoids (tetraterpenoids; a class of terpenoids) is a complicated process, and regulatory mechanisms in P. rhodozyma and other producers remain poorly known, although some research progress has been made during the past five years [16-20].

Farnesyl diphosphate (FPP) is a branch-point metabolite of terpenoids and steroids, and CRTE catalyzes formation of geranylgeranyl diphosphate (GGPP) from FPP. This step is generally regarded as the beginning of the carotenoid biosynthesis pathway, because crtE (also known as GGPP synthase gene) is part of the carotenogenic gene cluster in all bacteria investigated to date. crt $\mathrm{E}$ expression is strongly enhanced during fruit ripening, which is accompanied by massive carotenoid formation in Capsicum (family Solanaceae) [15]. Formation of phytoene through condensation of two GGPP molecules is catalyzed by phytoene- $\beta$-carotene synthase (PBS; encoded by $p b s$ gene), a bifunctional enzyme that also displays lycopene cyclase function in P. rhodozyma [21, 22]. In four subsequent dehydration steps catalyzed by phytoene dehydrogenase (encoded by $c r t \mathrm{I}$ ) [23], double bonds are introduced and lycopene is synthesized. Lycopene is transformed into $\beta$-carotene by introduction of ring structures at both ends of the molecule, catalyzed by PBS [22]. $\beta$-carotene is hydroxylated and oxidized to astaxanthin by astaxanthin synthase (encoded by ast) [24, 27].

Regulation of the astaxanthin biosynthesis pathway in $P$. rhodozyma appeared to occur mainly at the transcript level of carotenogenic genes [25-27], since astaxanthin overproduction was associated with greatly increased expression of these genes. Similar results were obtained for the carotenoid-producing fungi Neurospora crassa [28, 29], Phycomyces blakesleeanus [30-32], Mucor circinelloides [33, 34], and Fusarium fujikuroi [35, 36].

Marcoleta et al. observed that the expression of $\operatorname{crt} \mathrm{I}$, pbs, and ast in P. rhodozyma was inhibited by addition of after adding $20 \mathrm{~g} / \mathrm{L}$ glucose $(20 \mathrm{~g} / \mathrm{L})$ to compare with medium, that deplete of glucose [26]. They also showed that the expression $c r t \mathrm{I}, p b s$ and ast was enhanced by addition of ethanol. They concluded that the astaxanthin repression by glucose was regulated at the transcript level [26]. Pamela et al. reported that transcriptional corepressor complex Cyc8-Tup1 is involved in regulation of carotenogenesis by glucose [17]. For large-scale astaxanthin production by $P$. rhodozyma, use of high glucose concentration $(>100 \mathrm{~g} / \mathrm{L})$ is more practical, in view of the simplified industrial process and less required manpower. We previously established an astaxanthinoverproducing mutant strain of $P$. rhodozyma, termed MK19, by 1-methyl-3-nitro-1-nitrosoguanidine (NTG) and Co60 mutagenesis [37]. MK19 is the most glucosetolerant $P$. rhodozyma strain reported to date; it maintains its ability to synthesize astaxanthin at glucose concentrations as high as $110 \mathrm{~g} / \mathrm{L}$ without repressive effect. The regulatory mechanism by glucose in MK19 presumably differs from that in parental (wild-type; WT) strain JCM9042.

In the well-known species Saccharomyces cerevisiae (baker's yeast), regulation of glucose repression is fairly well understood, and the proteins involved have been studied extensively [38]; however, very little is known regarding the glucose signaling mechanism or factors involved in repressor complex structures under high- and low-glucose conditions. No studies along these lines have been performed in P. rhodozyma. Glucose repression occurs mainly at the transcriptional level. Accordingly, we investigated deregulation of astaxanthin synthesis by glucose at the transcriptional level and glucose signaling pathway level in JCM9042 and MK19 to clarify the underlying molecular mechanisms.

\section{Results}

MK19 is a glucose metabolic derepression strain

Both cell growth and astaxanthin synthesis in WT JCM9042 were inhibited by high glucose concentration. $\mathrm{Y}_{\mathrm{X} / \mathrm{S}}$ (biomass production (g) per $\mathrm{g}$ glucose) for JCM9042 was reduced from 0.42 at glucose concentration $40 \mathrm{~g} / \mathrm{L}$ to 0.218 at glucose concentration $110 \mathrm{~g} / \mathrm{L}$, and $\mathrm{Y}_{\mathrm{P} / \mathrm{S}}$ (astaxanthin production $(\mathrm{mg})$ per g glucose) was reduced by $>60 \%$ from $0.033(40 \mathrm{~g} / \mathrm{L})$ to $0.01(110 \mathrm{~g} /$ $\mathrm{L})$ (Table 1). In contrast, no such reduction of $\mathrm{Y}_{\mathrm{P} / \mathrm{S}}$ was observed for MK19.

Table 1 Biomass yield and astaxanthin production (per gram glucose) in JCM9042 and MK19

\begin{tabular}{|c|c|c|c|c|c|c|}
\hline \multirow{2}{*}{$\begin{array}{l}\text { Glucose } \\
(\mathrm{g} / \mathrm{L})\end{array}$} & \multicolumn{3}{|l|}{$Y_{X / S}(g / g)^{a}$} & \multicolumn{3}{|l|}{$\mathrm{Y}_{\mathrm{P} / \mathrm{S}}(\mathrm{mg} / \mathrm{g})^{\mathrm{b}}$} \\
\hline & 40 & 110 & 160 & 40 & 110 & 160 \\
\hline JCM9042 & $0.42 \pm 0.003$ & $0.218 \pm 0.009$ & $0.136 \pm 0.003$ & $0.033 \pm 0.003$ & $0.010 \pm 0.001$ & $0.003 \pm 0.001$ \\
\hline MK19 & $0.405 \pm 0.036$ & $0.300 \pm 0.004$ & $0.202 \pm 0.003$ & $0.250 \pm 0.004$ & $0.255 \pm 0.001$ & $0.073 \pm 0.006$ \\
\hline
\end{tabular}

${ }^{\mathrm{a}}$ biomass yield $(\mathrm{g})$ per g glucose. ${ }^{\mathrm{b}}$ astaxanthin production $(\mathrm{mg})$ per g glucose 
The low biomass yield in MK19 may have resulted from insufficient oxygen in flasks, leading to incomplete oxidation of glucose by aerobic respiration. Ethanol accumulation was high $(>9 \mathrm{~g} / \mathrm{L})$ for $110 \mathrm{~g} / \mathrm{L}$ glucose, but low for in $40 \mathrm{~g} / \mathrm{L}$ glucose. Because Crabtree effect can be inhibited by increasing dissolved oxygen level (DO), we reduced medium to $6 \%$ liquid volume in flask in order to increase DO. Under this condition, ethanol concentration was much lower than for $10 \%$ liquid volume (Table 2).

Biomass yield (cell growth) increased significantly at $6 \%$ liquid volume for $110 \mathrm{~g} / \mathrm{L}$ glucose; $\mathrm{Y}_{\mathrm{X} / \mathrm{S}}$ was 0.382 , close to the value for $40 \mathrm{~g} / \mathrm{L}$ glucose (Table 3). Maximal cell density $\left(\mathrm{OD}_{600}>70\right)$, was obtained at $4 \%$ liquid volume. At $6 \%$ liquid volume, astaxanthin production $\left(\mathrm{Y}_{\mathrm{P} / \mathrm{S}}\right)$ increased to $0.32 \mathrm{mg} / \mathrm{g}$ (Table 3) without change of astaxanthin content. These findings indicate that MK19 is a glucose metabolic derepression strain in regard to both astaxanthin production and cell growth, when DO is sufficient. We used $10 \%$ liquid volume for all subsequent experiments because astaxanthin content $(\mu \mathrm{g} / \mathrm{g}$ dry weight) did not differ for 6 vs. 10\% liquid volume.

\section{$\beta$-Carotene and astaxanthin synthesis in MK19 are not inhibited by high glucose}

We examined levels of total carotenoids and pigments other than astaxanthin in cultured JCM9042 and MK19, to investigate regulatory mechanisms of carotenoid biosynthesis by glucose. Total carotenoids and most other pigments were strongly inhibited by high $(110 \mathrm{~g} / \mathrm{L})$ glucose in JCM9042 throughout the fermentation process; in particular, $\beta$-carotene and astaxanthin levels at $72 \mathrm{~h}$ were less than $30 \%$ of levels at $40 \mathrm{~g} / \mathrm{L}$ glucose (Fig. 1a,b). $\beta$-carotene synthesis increased notably after $72 \mathrm{~h}$, when glucose supply was nearly exhausted (Fig. 1a). Levels of total pigments, $\beta$-carotene, and astaxanthin in JCM9042 were much higher for $40 \mathrm{~g} / \mathrm{L}$ than for $110 \mathrm{~g} / \mathrm{L}$ glucose, particularly after $48 \mathrm{~h}$ when glucose was nearly exhausted (Fig. 1a,b). These findings suggest that high glucose concentration in JCM9042 inhibited synthesis of $\beta$-carotene, astaxanthin, and other pigments. Despite notable accumulation of $\beta$-carotene, inhibition of astaxanthin synthesis continued even after exhaustion of

Table 2 Ethanol yield of MK19 as a function of time for various combinations of glucose concentration and liquid volume

\begin{tabular}{lllll}
\hline & Glucose concentration $(\mathrm{g} / \mathrm{L})$ & 40 & 110 & 110 \\
& Liquid volume & $10 \%$ & $10 \%$ & $6 \%$ \\
\hline Time (h) & 0 & 0 & 0 & 0 \\
& 24 & 0.03 & 0.244 & 0.046 \\
48 & 0.138 & 0.807 & 0.146 \\
72 & 0 & 6.729 & 0.811 \\
96 & 0 & 9.104 & 1.317 \\
\hline
\end{tabular}

Table 3 Maximal biomass yield $\left(Y_{X / S}\right)$ and astaxanthin production ( $\left.Y_{P / S}\right)$ in MK19 for various combinations of glucose concentration and liquid volume

\begin{tabular}{llll}
\hline Glucose concentration & Liquid volume & $Y_{X / S}(\mathrm{~g} / \mathrm{g})$ & $Y_{P / S}(\mathrm{mg} / \mathrm{g})$ \\
\hline $40 \mathrm{~g} / \mathrm{L}$ & $10 \%$ & $0.405 \pm 0.036$ & $0.25 \pm 0.004$ \\
$40 \mathrm{~g} / \mathrm{L}$ & $6 \%$ & $0.420 \pm 0.016$ & $0.275 \pm 0.026$ \\
$110 \mathrm{~g} / \mathrm{L}$ & $10 \%$ & $0.300 \pm 0.004$ & $0.255 \pm 0.001$ \\
$110 \mathrm{~g} / \mathrm{L}$ & $6 \%$ & $0.382 \pm 0.007$ & $0.320 \pm 0.029$ \\
\hline
\end{tabular}

glucose, indicating differing regulatory mechanisms of the two pigments by glucose.

Transfer of precursors (e.g., GGPP, phytoene) to carotenoids was limited at glucose concentrations $>40 \mathrm{~g} / \mathrm{L}$. The reduction of $\beta$-carotene (6- to 7 -fold) observed for high vs. low glucose from 24 to $72 \mathrm{~h}$ was much greater than that of total carotenoids (2- to 3-fold) (Fig. 1). Thus, $\beta$-carotene synthesis appears to be the ratelimiting step for astaxanthin synthesis at high glucose concentration.

In stationary phase of growth in MK19, high glucose $(110 \mathrm{~g} / \mathrm{L})$ caused slight enhancement rather than repression of total carotenoids $(1231 \mu \mathrm{g} / \mathrm{g}(\mathrm{DW})$ vs. $937 \mu \mathrm{g} /$ $\mathrm{g}(\mathrm{DW})$ for $40 \mathrm{~g} / \mathrm{L}$ glucose) and astaxanthin $(900 \mu \mathrm{g} /$ g(DW) vs. $790 \mu \mathrm{g} / \mathrm{g}(\mathrm{DW})$ ) (Fig. 1c,d). At $40 \mathrm{~g} / \mathrm{L}$ glucose, MK19 had astaxanthin level strike greater than that of JCM9042, but lower $\beta$-carotene level (Fig. 1), indicating that efficiency of astaxanthin synthase (AST) activity for conversion of $\beta$-carotene to astaxanthin was high in MK19 and low in JCM9042. For $110 \mathrm{~g} / \mathrm{L}$ glucose in comparison to $40 \mathrm{~g} / \mathrm{L}$ glucose, astaxanthin synthesis was slower during lag and log phases, and excessive astaxanthin was accumulated in stable phase, but final astaxanthin content was similar (Fig. 1c,d). Accumulation of $\beta$-carotene was strikingly higher (up to $>50$-fold) for $110 \mathrm{~g} / \mathrm{L}$ vs. $40 \mathrm{~g} / \mathrm{L}$ glucose. Taken together, these findings suggest that (i) additional AST is required to synthesize more astaxanthin in spite of high ast gene expression; (ii) $\beta$-carotene synthesis is completely deregulated by high glucose level; (iii) inhibition of astaxanthin synthesis is relieved when glucose is exhausted.

In conclusion, inhibition of astaxanthin synthesis could be more strongly relieved in MK19 than in WT, and such relief resulted from enhanced expression of ast gene (Miao et al. 2011). Deregulation of $\beta$-carotene synthesis and relief of astaxanthin regulation by glucose lead to excessive $\beta$-carotene accumulation in MK19, and further increase of astaxanthin content requires enhancement of ast activity.

\section{Expression of carotenogenic genes pbs and ast is derepressed in MK19}

The pigment profiles described above clearly indicate that repression of astaxanthin biosynthesis pathway by 

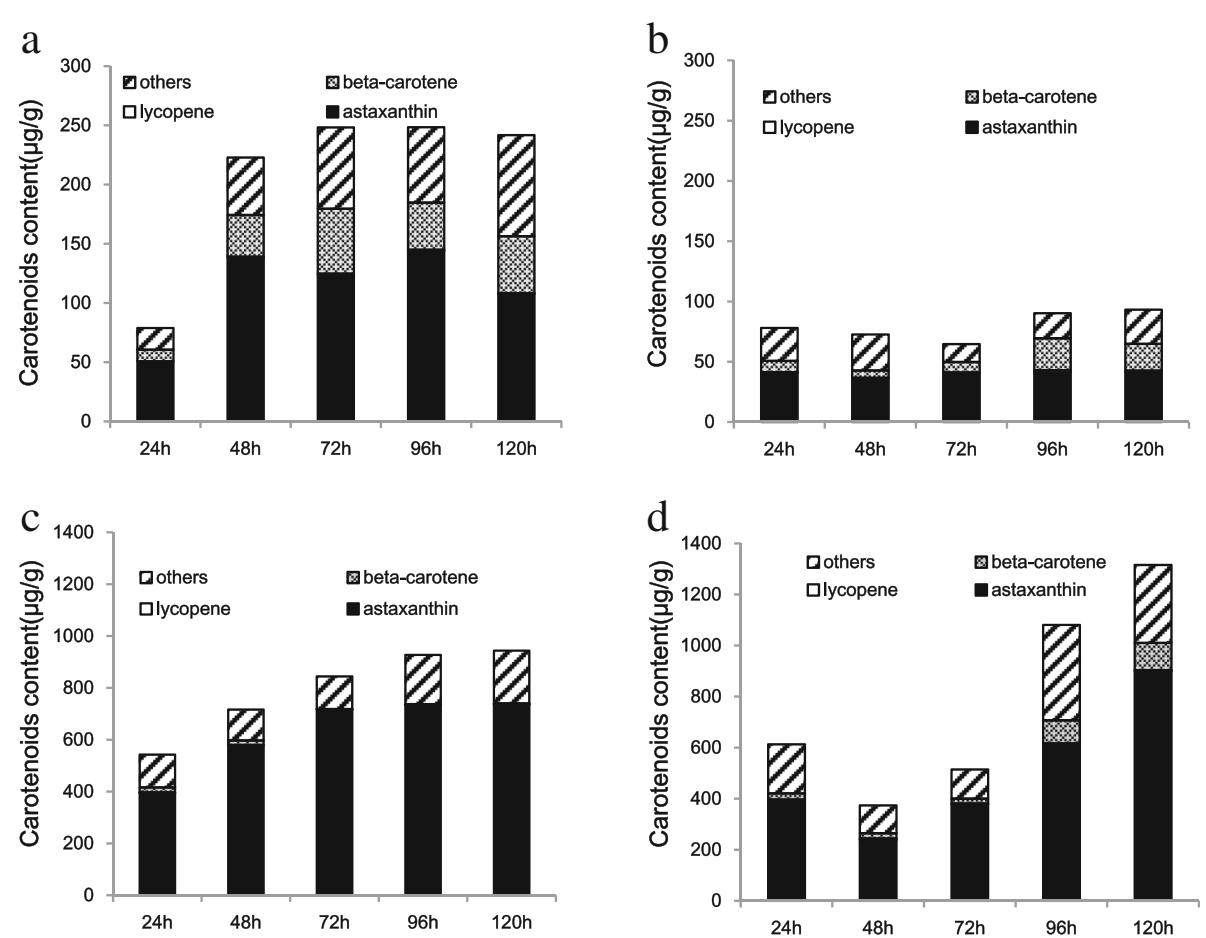

Fig. 1 Carotenoid profiles of WT JCM9042 at $40 \mathrm{~g} / \mathrm{L}$ glucose (a), JCM9042 at $110 \mathrm{~g} / \mathrm{L}$ glucose (b), mutant MK19 at $40 \mathrm{~g} / \mathrm{L}$ glucose (c), and MK19 at $110 \mathrm{~g} / \mathrm{L}$ glucose $(\mathbf{d})$

glucose occurs primarily at the lycopene-to- $\beta$-carotene reaction step, and to a lesser degree at the $\beta$-caroteneto-astaxanthin step in JCM9042. The large reduction of total pigment level under high glucose in JCM9042 suggests that astaxanthin synthesis flux may be blocked at the beginning of carotenoid synthesis; e.g., FPP-to-GGPP or GGPP-to-phytoene step. The deregulation of astaxanthin synthesis by high glucose in MK19 appears to be due mainly to uncontrolled generation of $\beta$-carotene from lycopene. To test these possibilities and investigate the molecular mechanisms underlying inhibition of astaxanthin synthesis in P. rhodozyma, we examined expression of carotenogenic genes under high vs. low glucose concentration in JCM9042 and MK19.

Expression of carotenogenic genes was fairly stable throughout cultivation at $110 \mathrm{~g} / \mathrm{L}$ glucose except for ast, which showed increased mRNA until $48 \mathrm{~h}$ (Fig. 2). Therefore, a positive effect of ethanol on transcription of carotenogenic genes can be ruled out (Table 2) [26], and we used only log phase $(48 \mathrm{~h})$ samples from 40 and 160 $\mathrm{g} / \mathrm{L}$ glucose conditions for comparisons of gene expression.

JCM9042 showed much lower mRNA level for structural gene ast at $110 \mathrm{~g} / \mathrm{L}$ than $40 \mathrm{~g} / \mathrm{L}$ glucose (Fig. 2b), consistently with the findings of Marcoleta et al. [26], and mRNA level at $72 \mathrm{~h}$ under $110 \mathrm{~g} / \mathrm{L}$ glucose was $30 \%$ of that at $48 \mathrm{~h}$. This finding may account in part for the observation that astaxanthin level did not increase even after exhaustion of glucose (72 h) (Fig. 1), and the low proportion $(40 \%)$ of astaxanthin among total carotenoids in JCM9042 at $110 \mathrm{~g} / \mathrm{L}$ glucose. In MK19, glucose concentration did not notably affect ast expression except at $24 \mathrm{~h}$. In both JCM9042 and MK19, crtI transcription was higher at 110 than at $40 \mathrm{~g} / \mathrm{L}$ glucose, but was reduced at $160 \mathrm{~g} / \mathrm{L}$ glucose (Fig. 2a). In contrast, Marcoleta et al. [26] found that $c r t$ I was downregulated even at low glucose concentration. In JCM9042 at $110 \mathrm{~g} / \mathrm{L}$, enhancement of $c r t \mathrm{I}$ mRNA was correlated with accumulation of lycopene, whose production is catalyzed by the enzyme phytoene desaturase (CrtI).

In JCM9042, mRNA transcription of $c r t \mathrm{E}$ and $p b s$ declined rapidly as glucose concentration increased $(>40$ $\mathrm{g} / \mathrm{L}$ ), suggesting that repression of astaxanthin by glucose occurs mainly during synthesis of GGPP, or of phytoene and lycopene. This concept is supported by the pigment profile of JCM9042, the reduction of total pigment level during cultivation, and the low proportion of $\beta$-carotene from 24 to $72 \mathrm{~h}$ at $110 \mathrm{~g} / \mathrm{L}$ glucose, when glucose is still abundant. In MK19, pbs was completely deregulated by glucose (Fig. 2d). crtE expression was inhibited by high glucose in MK19, even though crtE mRNA level was $>2$-fold higher than that of JCM9042 (Fig. 2c). This finding may account for the slow synthesis of total carotenoids in MK19 at $110 \mathrm{~g} / \mathrm{L}$ glucose prior to $\log$ phase. The enhanced crtE mRNA level in MK19 relieved the inhibition of precursor-to-pigment synthesis, 

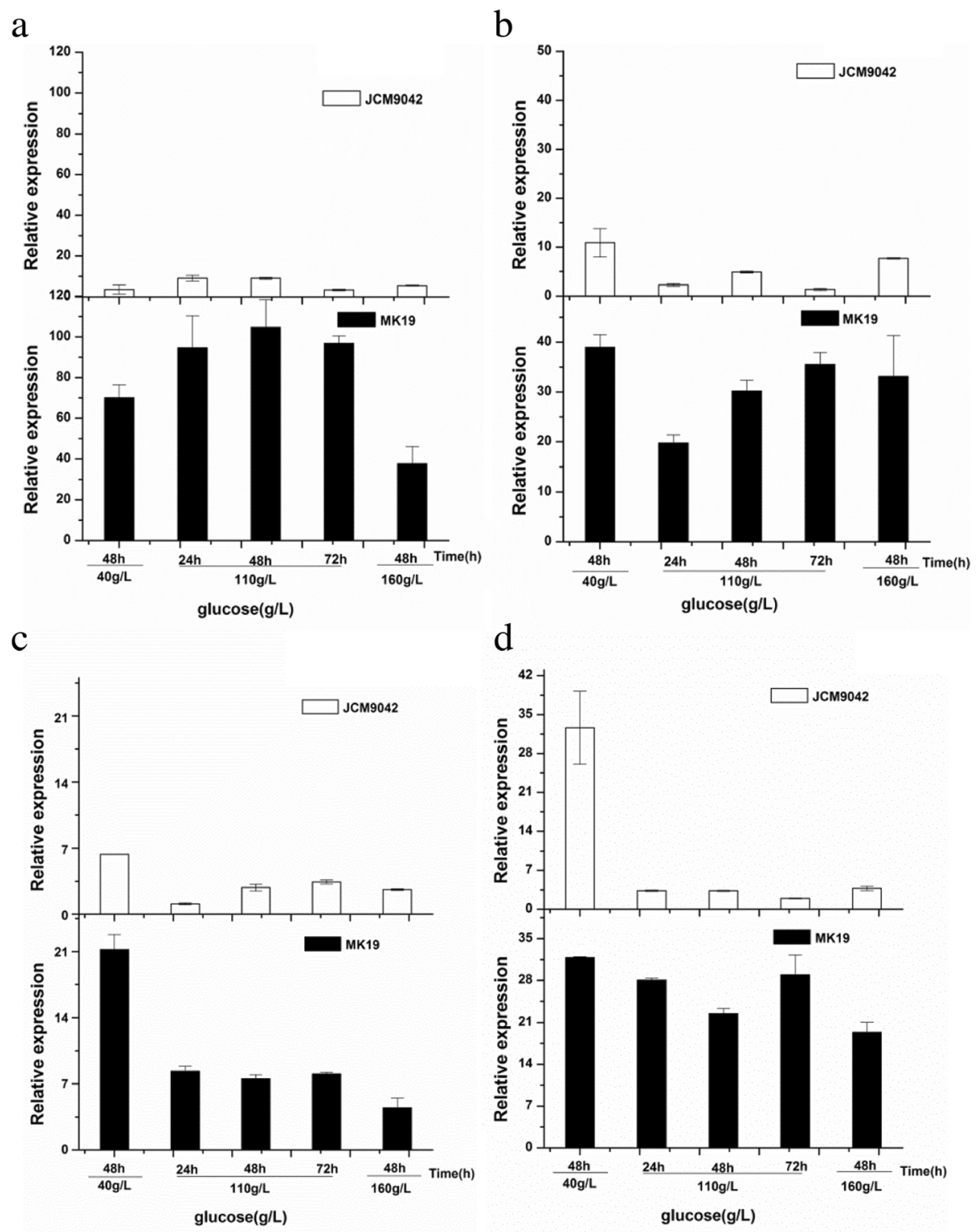

Fig. 2 Relative expression of $\mathrm{crtl}(\mathbf{a})$, ast (b), crtE (c), and pbs (d) genes at glucose concentrations of 40, 110, and $160 \mathrm{~g} / \mathrm{L}$

and in combination with deregulation of $p b s$ gene resulted in final astaxanthin level similar to that at low glucose. It is also suggested that further increase of $c r t \mathrm{E}$ mRNA may further enhance astaxanthin content. Uncontrolled astaxanthin synthesis at high glucose was presumably due mainly to deregulation of $p b s$.

creA expression is not induced by high glucose in MK19 Carotenogenic genes are regulated by glucose through protein CreA, a global negative regulator. We examined CreA expression to evaluate the possibility that astaxanthin synthesis is controlled by glucose through downregulation of pbs expression. The role of creA has been intensively was studied in S. cerevisiae [38]. Moreno et al. [38] found that high glucose induced creA gene expression in S. cerevisiae. Cifuentes et al. [16] showed that carotenogenic synthesis gene promoters contained a CreA binding position, and used electrophoretic mobility shift assays (EMSAs) to improve the interactions of these genes with CreA. In both JCM9042 and MK19, creA mRNA was at a similar low level when glucose was exhausted after $48 \mathrm{~h}$ culture. In JCM9042 at $110 \mathrm{~g} / \mathrm{L}$ glucose, creA expression was strongly induced at $60 \mathrm{~min}$, whereas creA mRNA in MK19 remained at a similar low level regardless of glucose induction time (Fig. 3). 


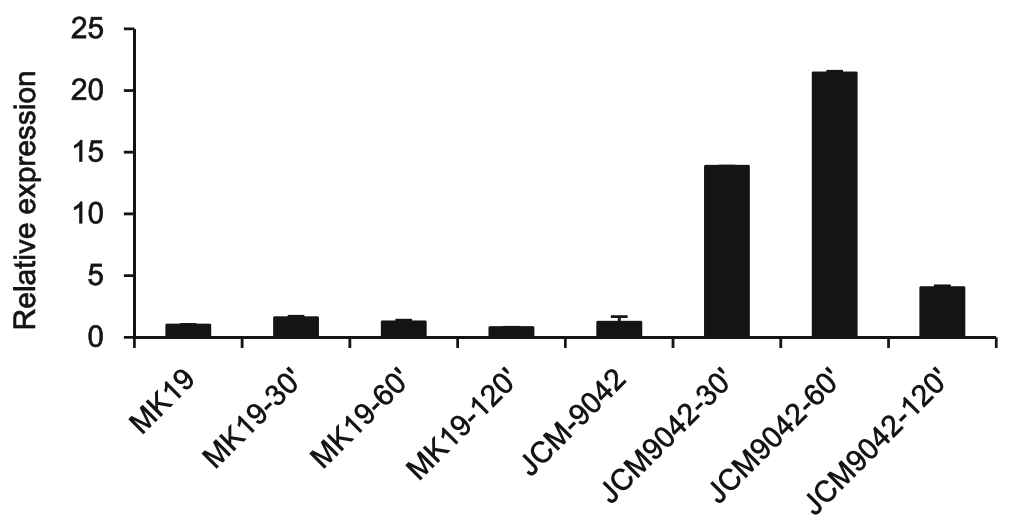

Fig. 3 Relative expression of CreA in MK19 and JCM9042 at glucose induction times 0, 30, 60, and 120 min as indicated in the X-axis captions

Expression of alcA, $c r t \mathrm{E}$, and $p b s$ at glucose induction time $30 \mathrm{~min}$ was suppressed in JCM9042 (Fig. 4a), but enhanced in MK19 (Fig. 4b). Thus, deregulation by glucose of carotene synthesis genes in MK19 appears to result from failure induction of the global negative regulator CreA.

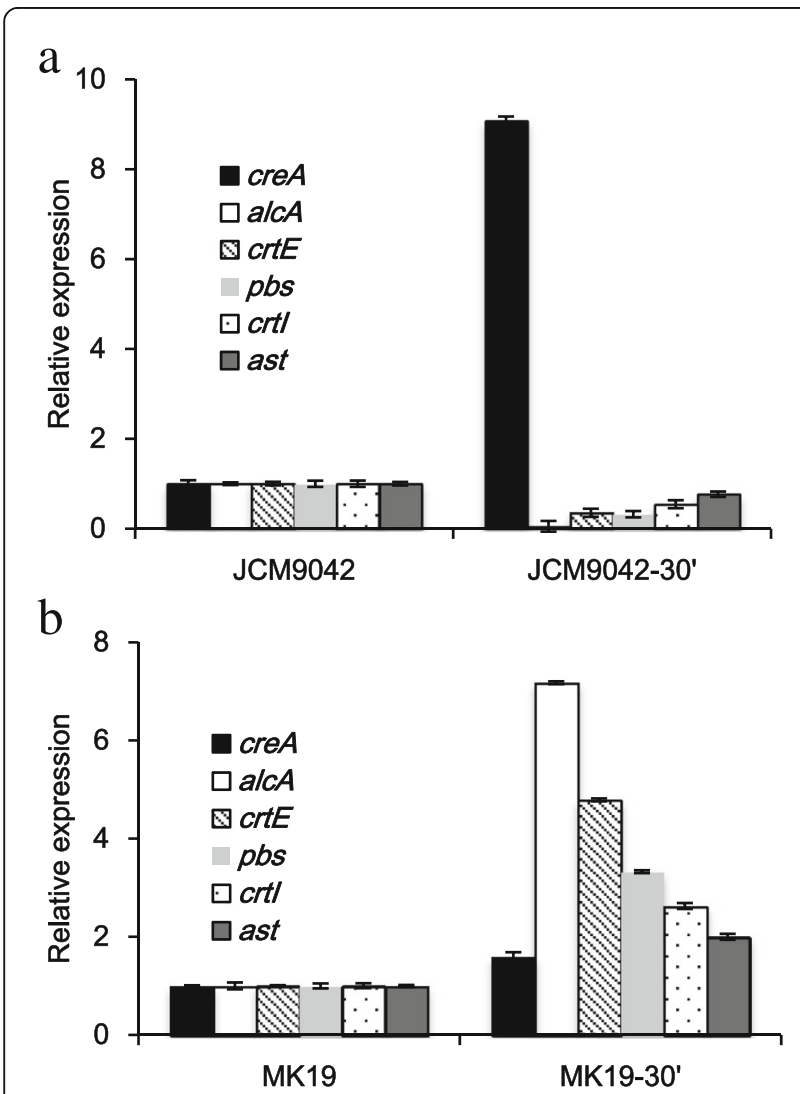

Fig. 4 Relative expression of creA, alcA, and carotenoid synthesis genes in JCM9042 (a) and MK19 (b) at glucose induction times 0 and $30 \mathrm{~min}$. Expression at $48 \mathrm{~h}$ was defined as 1. alcA, encoding the alcohol dehydrogenase I and repressed by creA, was used as control
To test the concept that derepression of astaxanthin synthesis genes such as $p b s$ in MK19 resulted mainly from low creA mRNA, we expressed extra copies of this gene. The transformed clone MK19-CreA9 (termed "mutant 9") showed a distinct pink color, particularly at early culture stages (before $72 \mathrm{~h}$ ), when glucose was abundant, even though initial glucose concentration was low $(40 \mathrm{~g} / \mathrm{L}$ in seed medium, the mutants cannot grow in synthesized medium.). Total carotenoid content of mutant 9 was only $\sim 50 \%$ that of MK19 (Fig. 5b). In comparison with MK19, mutant 9 showed slower cell growth prior to $72 \mathrm{~h}$, and much lower expression of $p b s$, ast, crtE, crtI, and alc $\mathrm{A}$ genes (Fig. 5a).

These findings indicate that derepression by glucose of astaxanthin synthesis in MK19 was due to the fact that creA was not induced by glucose, and consequently did not inhibit expression of its target genes ( $p b s$, ast, etc.).

\section{Expression of reg 1 and $h \times k 2$ in MK19 glucose signaling pathway genes was not induced by glucose}

We measured expression of glucose signaling pathway genes (snf1, snf4, sip5, sak1, glc7, hxk2, reg1) to elucidate the molecular mechanisms underlying regulation of astaxanthin synthesis by glucose in $P$. rhodozyma. The protein CreA is regulated by Snf1 kinases and Glc phosphatase. At high glucose level, Snf1 is inactive and cannot phosphorylate CreA. When glucose level falls, Snf1 becomes active and phosphorylates CreA, which cannot then bind to target genes such as alc $\mathrm{A}$ and carotenogenic genes. High glucose also inactivates Snf1 by stimulating activity of Glc7-Reg1 phosphatase. Presence of Hxk2 in the repressor complex is necessary for inhibition of Snf1 kinase-induced CreA phosphorylation [38]. Addition of glucose promotes Hxk2/ Reg1 interaction and inactivation of Snf1. $h x k 2$ mRNA level in MK19 was much lower than in JCM9042, and $h x k 2$ expression was not induced by glucose (Fig. 6). This is one reason why MK19 was unable to maintain CreA in active state and 

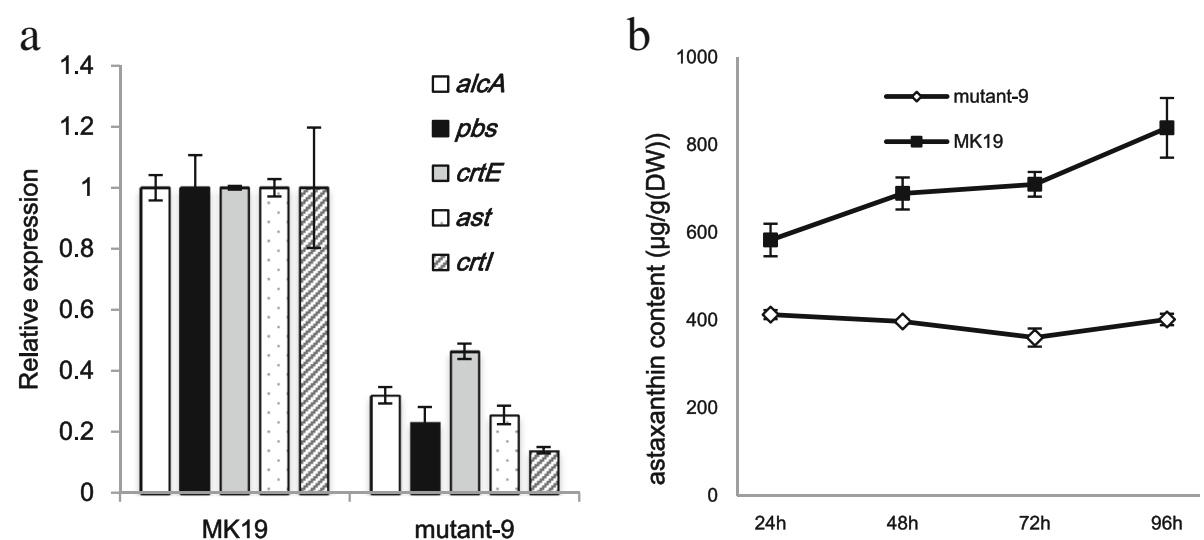

Fig. 5 (a) Relative expression of alcA and carotenoid synthesis genes in MK19 and MK19-CreA9 (mutant 9) at $24 \mathrm{~h}$ at $40 \mathrm{~g} / \mathrm{L}$ glucose. Expression in MK19 was defined as 1. (b) Astaxanthin content of MK19 and mutant 9 as a function of fermentation time

showed downregulation of carotenogenic genes at high glucose level. In JCM9042 at $110 \mathrm{~g} / \mathrm{L}$ glucose, $r e g 1$ mRNA level was much higher $(\sim 10$-fold $)$ at glucose induction time $30 \mathrm{~min}$ than at $0 \mathrm{~min}$. In contrast, reg 1 mRNA level in MK19 was only slightly higher at $30 \mathrm{~min}$ than at 0 min (Fig. 6). sak1 gene expression, which promotes Snf1 kinase activity at low glucose level, was induced by glucose in MK19. These findings indicate that failure of glucose to induce $h x k 2$ and reg 1 explains why astaxanthin synthesis in MK19 was not inhibited at high glucose level.

\section{Discussion}

Phaffia rhodozyma is a preferred industrial strain for production of natural astaxanthin because of several desirable properties, e.g., synthesis of free astaxanthin as principal carotenoid in the absence of light, fermentation of many types of saccharides, and high cell density in autofermentors. Typically, cell growth and astaxanthin synthesis of $P$. rhodozyma, which displays positive Crabtree effect, are inhibited by high glucose concentration. We previously established a moderate-temperature, high-glucose-tolerant, astaxanthin-overproducing $P$. rhodozyma strain, termed MK19 [37]. Astaxanthin synthesis by MK19 is not repressed by high glucose level (up to $110 \mathrm{~g} / \mathrm{L}$ ). In contrast, cell growth and carotenoid production of WT strain JCM9042 can only tolerate glucose level up to $40 \mathrm{~g} / \mathrm{L}$. MK19 is a glucose metabolic derepression strain in regard to both astaxanthin production and cell growth, when DO is sufficient. $h x k 2$ mRNA level in MK19 is low (see Results), consistent with the previous finding of reduced Crabtree effect in an $h x k 2$ mutant of $S$. cerevisiae, resulting in nearly complete respiratory metabolism at high glucose level [38].

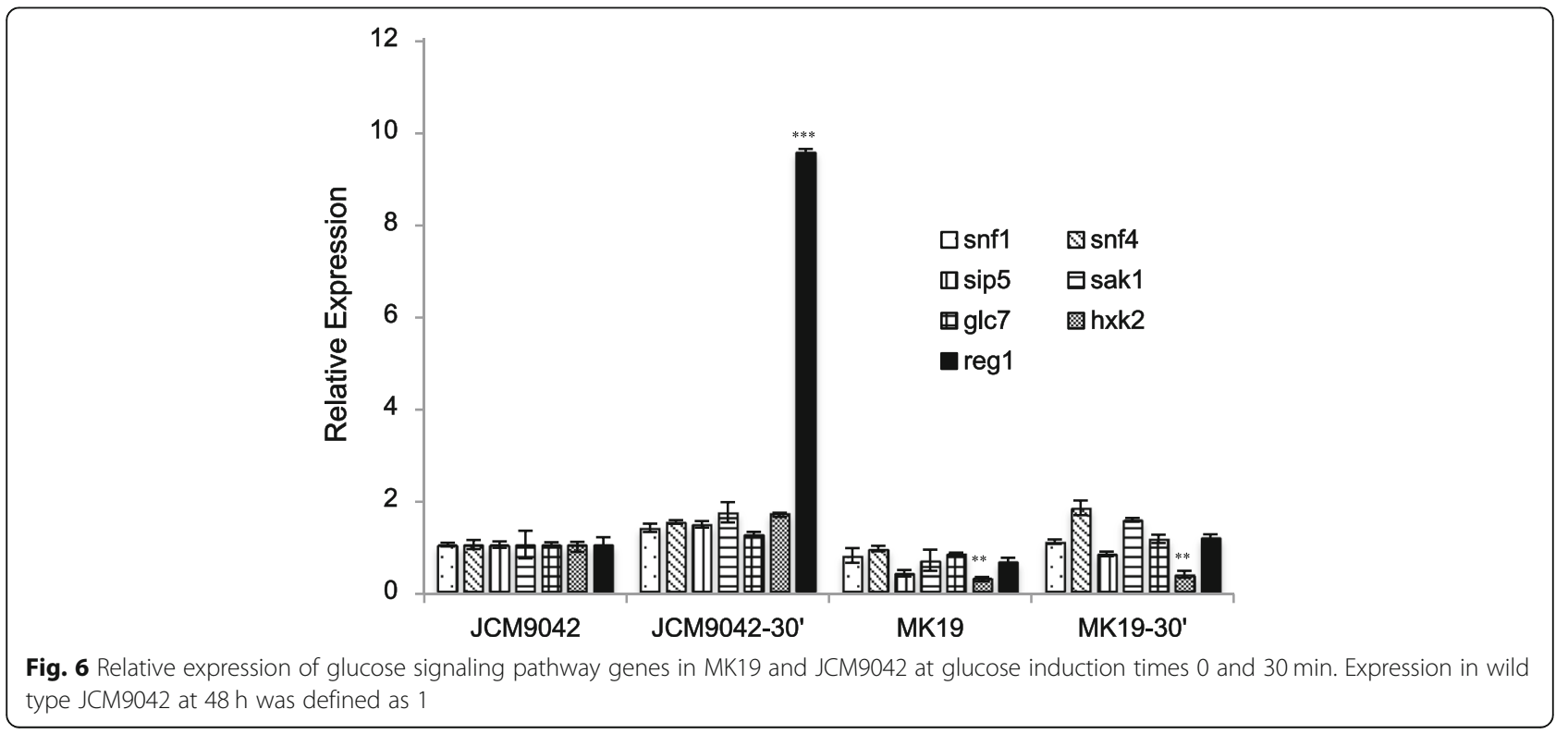


Repression of astaxanthin synthesis by high $(110 \mathrm{~g} / \mathrm{L})$ glucose in $P$. rhodozyma depends mainly on bifunctional enzyme phytoene- $\beta$-carotene synthase (PBS)

The reduced levels of $\beta$-carotene, astaxanthin, and total pigments observed at $110 \mathrm{~g} / \mathrm{L}$ glucose in JCM9042 indicate that repression occurs at the lycopene-to- $\beta$-carotene step, $\beta$-carotene-to-astaxanthin step, or even beginning of the carotenoid biosynthesis pathway (GGPP formation). Levels of $\beta$-carotene and total carotenoids were much lower (11-14\%) at 110 than at $40 \mathrm{~g} / \mathrm{L}$ glucose, suggesting that $\beta$-carotene synthesis is the rate-limiting step for astaxanthin synthesis at high glucose level. In contrast, synthesis of total carotenoids and astaxanthin was not controlled by high glucose during MK19 stable phase. At $40 \mathrm{~g} / \mathrm{L}$ glucose, astaxanthin content was 10fold higher whereas $\beta$-carotene was much lower in MK19 than in JCM9042, indicating that $\beta$-carotene-toastaxanthin conversion efficiency was high in MK19 but very low in JCM9042. In MK19, $\beta$-carotene synthesis was not inhibited by $110 \mathrm{~g} / \mathrm{L}$ glucose; final astaxanthin content was similar at 40 vs. $110 \mathrm{~g} / \mathrm{L}$ glucose, and $\beta$ carotene accumulation at $120 \mathrm{~h}$ was $\sim 50$-fold higher at $110 \mathrm{~g} / \mathrm{L}$ glucose. Additional AST activity was evidently necessary to further increase astaxanthin production, even though ast is highly expressed in MK19 [27]. Metabolic engineering (overexpression) of ast is a useful approach for further enhancing astaxanthin production in MK19 [39]. These conclusions are consistent with observed expression of structural genes (crtE, pbs, ast, $c r t \mathrm{I})$ in JCM9042 and MK19 at various glucose concentrations (Fig. 2).

Transcription of $c r t \mathrm{E}$ and $p b s$ in JCM9042 was greatly inhibited at glucose concentrations $>40 \mathrm{~g} / \mathrm{L}$; in particular, $p b s$ was reduced to $10 \%$ at $110 \mathrm{~g} / \mathrm{L}$ glucose. These findings suggest that repression of astaxanthin by glucose metabolites in P. rhodozyma also occurs at the GGPP, phytoene, and lycopene synthesis steps. This concept is supported by the consistency between expression profiles and pigment profiles in JCM9042.

Although crtE mRNA level was 2-fold higher in MK19 than in JCM9042, this gene was still inhibited by high glucose, resulting in slower accumulation of pigments prior to log phase. Final total pigment content was higher at 110 than at $40 \mathrm{~g} / \mathrm{L}$ glucose, even though $\operatorname{crt} \mathrm{E}$ mRNA level was lower at $40 \mathrm{~g} / \mathrm{L}$ glucose. GGPP is a point metabolite that connects carotenoids to other isoprenoids. High carotenoid synthesis, stimulated by highglucose-induced deregulation of $p b s$ and ast, presumably redistributes GGPP from other isoprenoids biosynthesis pathways to carotenoid flux pathway in MK19, leading eventually to similar final astaxanthin content at low and high glucose levels. We conclude that uncontrolled astaxanthin synthesis at high glucose in MK19 results mainly from deregulation of $p b s$ and (to a lesser degree) ast gene, leading to high transfer efficiency from GGPP to phytoene, lycopene to $\beta$-carotene, and $\beta$-carotene to astaxanthin.

\section{Repression of $p b s$ by glucose is mediated by creA, reg1, and $h \times k 2$ in the glucose signaling pathway}

CreA, the transcription factor primarily responsible for repression of genes necessary for utilization of alternative fermentable carbon sources, binds to DNA and inhibits transcription of target genes. Snf1 kinase plays a key role in regulation of the glucose repression signaling pathway. The relationships among glucose level, Snf1 kinase activity, CreA phosphorylation, Glc7-Reg1 phosphatase, alcA, carotenogenic genes, and Hxk2/ Reg1 interaction are described in Results/ "Expression of reg1 and $h x k 2$ in MK19 glucose signaling pathway genes ...". Upregulation of genes with CreA binding sites has been reported in $h x k 2$-deletion strains [38].

The role of creA has been intensively studied in S. cerevisiae [38]. In both JCM9042 and MK19, creA mRNA was at similar low levels when glucose was exhausted after $48 \mathrm{~h}$ culture, whereas its level was strikingly different in the two strains at $110 \mathrm{~g} / \mathrm{L}$ glucose with $60 \mathrm{~min}$ induction time (Fig. 3). At 30 min glucose induction time, JCM9042 showed repression of alcA, crtE, and $p b s$, together with strong induction of $c r e \mathrm{~A}$, whereas MK19 did not show creA induction or repression of its target genes. Thus, deregulation by glucose of carotenoid synthesis genes in MK19 is evidently due to failure of global negative regulator CreA. This concept is supported by the observed downregulation of alc $\mathrm{A}, c r t \mathrm{E}, p b s$, and ast in creA-overexpressing strain MK19-CreA9 (mutant 9).

To clarify the molecular mechanisms underlying regulation of astaxanthin synthesis by glucose in JCM9042 and MK19, we measured expression of glucose signaling pathway genes as described under Results/ "Expression of reg1 and $h x k 2$ in MK19 glucose signaling pathway genes". $h x k 2$ mRNA level was much lower in MK19 than in JCM9042, and was not induce by glucose (Fig. 6). reg1 mRNA level in JCM9042 at $110 \mathrm{~g} / \mathrm{L}$ glucose was $\sim 10$ fold higher at induction time $30 \mathrm{~min}$ than at $0 \mathrm{~min}$, but in MK19 was only slightly higher at $30 \mathrm{~min}$ than at 0 min. Low $h \times k 2$ and reg1 mRNA levels accounted for the failure of MK19 to maintain CreA active state, and for downregulation of carotenogenic genes at high glucose concentration. In contrast, expression of $s a k 1$, which promotes Snf1 kinase activity when glucose is exhausted, was enhanced in MK19 at $110 \mathrm{~g} / \mathrm{L}$ glucose. Thus, failure of glucose to induce $h \times k 2$ and reg1 accounts for the fact that astaxanthin synthesis in MK19 was not repressed at high glucose.

This study reports the involvement of reg 1 and $h x k 2$ in regulation of carotenogenesis in yeast. A proposed 
regulatory pattern of glucose signaling pathway on $p b s$ in JCM9042 and MK19 is shown schematically in Fig. 7.

The bifunctional enzyme PBS catalyzes generation of phytoene and $\beta$-carotene. Secondary metabolites (e.g., carotenoids) often display high synthesis/ accumulation in adverse (nutritionally deficient) environments. A simple method whereby microorganisms can reduce flux of secondary metabolites is to control multifunctional enzymes responsible for multiple pathway steps under good-nutrition conditions. Glucose at high concentration controls astaxanthin synthesis in P. rhodozyma primarily by inducing expression of glucose signaling pathway genes $(r e g 1, h x k 2)$ that maintain activity of creA and repress its target gene $p b s$. Thus, engineering of $p b s$, creA, $r e g 1$, and/or $h x k 2$ is potentially an efficient approach for increasing astaxanthin production on an industrial scale.

In summary, repression by glucose of astaxanthin in $P$. rhodozyma occurred mainly in the pathway steps for generation of GGPP, phytoene, lycopene, and astaxanthin, and was based on control of pbs, ast, and crtE transcription. Derepression of astaxanthin synthesis at high glucose level in astaxanthin-overproducing strain MK19 was due mainly to deregulation of $p b s$, ast, and glucose signaling genes $r e g 1, h \times k 2$, and creA, leading to enhanced efficiency of GGPP-to-phytoene and lycopeneto-astaxanthin transfers.

\section{Methods}

\section{Strains and culture conditions}

WT P. rhodozyma strain JCM9042 was obtained from the Institute of Physical and Chemical Research (RIKEN), Wako, Japan. The highly pigmented mutant strain MK19 was established by NTG and Co60 mutagenesis in our laboratory (Miao et al. 2010). Both strains were maintained on potato dextrose agar slants at $4{ }^{\circ} \mathrm{C}$.

Seed medium and fermentation medium were prepared as described previously [27]. Experiments were performed in shaking flask culture, with 250 -mL flasks

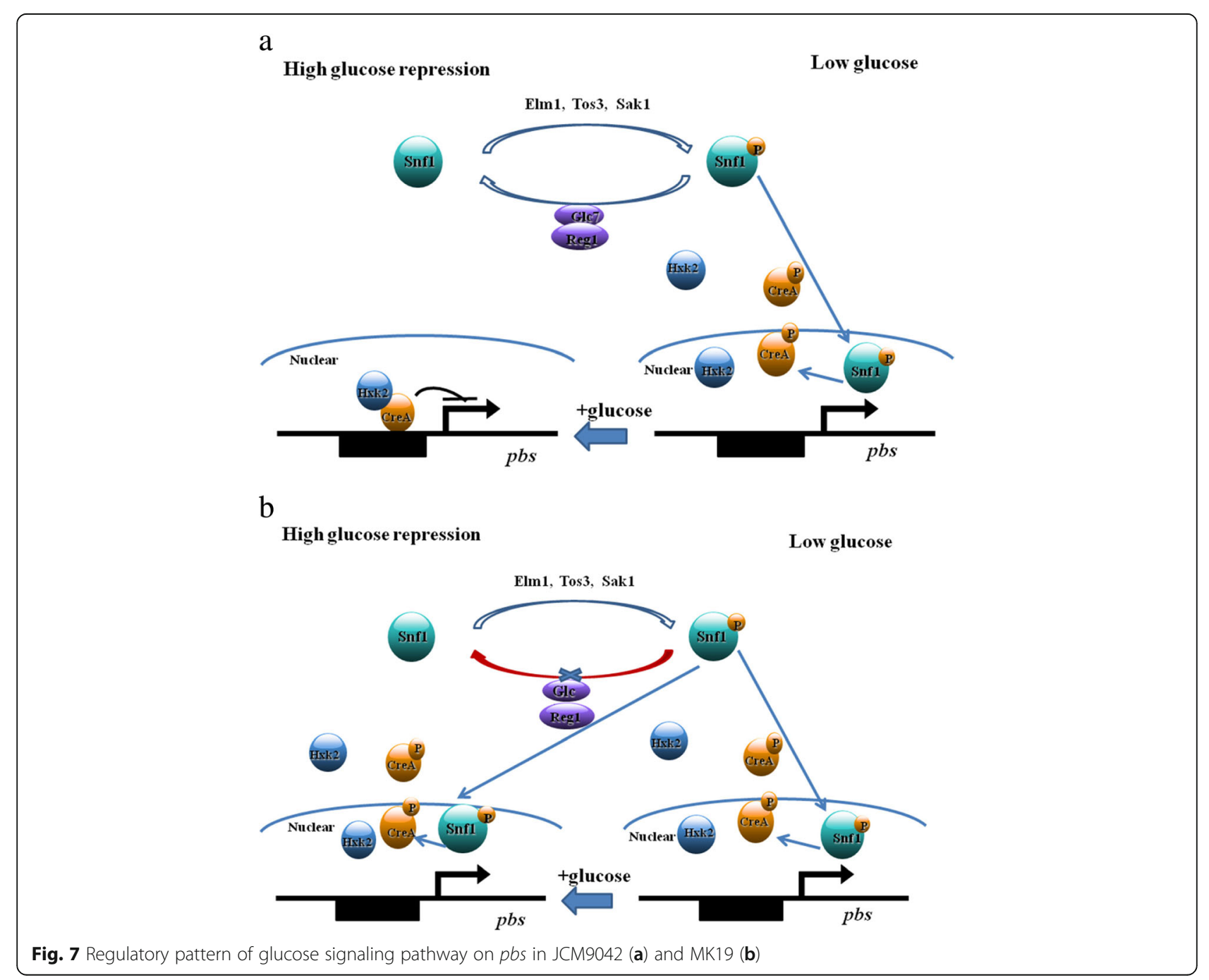


containing liquid volume $25 \mathrm{~mL}$. YPD medium:Glucose $20 \mathrm{~g} / \mathrm{L}$, yeast extracts $10 \mathrm{~g} / \mathrm{L}$, peptone $20 \mathrm{~g} / \mathrm{L}$.

WT and mutant cells were transferred from $4{ }^{\circ} \mathrm{C}$ slants to fresh slants, and kept for $72 \mathrm{~h}$ at $21-24{ }^{\circ} \mathrm{C}$. Loopfuls of lawn were inoculated to seed medium, and incubated for $72 \mathrm{~h}$ at $21-24^{\circ} \mathrm{C}$. Starter culture was produced by inoculation of $5 \%$ pre-incubation broth for an additional $36 \mathrm{~h}$. Overall production period of fermentation culture was 4-6 days on a rotary shaker $(210 \mathrm{rpm}), 21^{\circ} \mathrm{C}$, with samples taken at 12 -h or 24 -h intervals. Experiments were performed in triplicate or quadruplicate.

Cell concentration was estimated as $\mathrm{OD}_{600}$. Dry weight of cells was determined by centrifuging $35 \mathrm{~mL}$ broth at $12,000 \mathrm{rpm}$, rinsing with distilled water, and drying at $85^{\circ} \mathrm{C}$ to constant weight ( $\left.15 \mathrm{~h}\right)$.

\section{Measurement of astaxanthin}

$1 \mathrm{~mL}$ broth was centrifuged at $12,000 \mathrm{rpm}$ for $1 \mathrm{~min}$, and washed with distilled water. Pellets were mixed with $200 \mu \mathrm{L}$ dimethyl sulfoxide preheated to $70^{\circ} \mathrm{C}$, stirred, and the mixture was maintained at $70^{\circ} \mathrm{C}$ in a waterbath for $20 \mathrm{~min}$. Broken cells were extracted with methanol/ dichloromethane (3:1), agitated, and centrifuged at 2000 rpm. Supernatant was transferred to another tube. This process was repeated until pellets showed no red color. Astaxanthin and free ergosterol were analyzed quantitatively by HPLC on a C18 column $(250 \times 4.6 \mathrm{~mm} ; 5 \mu \mathrm{m}$; Chuangxintongheng Science \& Technology Co., Beijing): temperature $40^{\circ} \mathrm{C}$, flow rate $1.0 \mathrm{~mL} / \mathrm{min}$, wavelength $476 \mathrm{~nm}$ for astaxanthin or $280 \mathrm{~nm}$ for ergosterol. Mobile phase consisted of methanol 97\%, water $3 \%$. Astaxanthin and ergosterol were identified based on retention time relative to standard astaxanthin (Sigma).

\section{Total RNA purification, reverse transcription, and real- time PCR (RT-PCR)}

Total RNA purification and reverse transcription were performed as described previously [27, 37]. RT-PCR analyses were performed using an ABI 7900HT system (Applied Biosystems; Norwalk, CT, USA) with RNA samples as template. Dissociation curves were constructed to confirm amplification. Target genes were from NCBI (ncbi.nlm.nih.gov); database accession numbers and corresponding primer sets in RT-PCR were as described previously [27]. actin was used as control gene. Relative gene expression was calculated by $2^{-\triangle \triangle C T}$ (cycle threshold) method using Sequence Detection System software program v1.2.2 (Applied Biosystems). Each RT-PCR analysis was run in triplicate or quadruplicate to test consistency.

\section{Construction of MK19 creA-overexpressing strain}

Plasmid for expression of creA was constructed in pGBKT7 and transformed to MK19 according to Chi et al. [39].
$40 \mu \mathrm{g} / \mathrm{ml} \mathrm{G418} \mathrm{in} \mathrm{YPD} \mathrm{medium} \mathrm{was} \mathrm{used} \mathrm{to} \mathrm{screen}$ positive transformants. Plasmids of each transfromant were extracted using a yeast plasmid extraction kit (Tiangen Biotech; Beijing, China) and identified by PCR using primers creAP (5' -AAGACCTTGACATGA TTTTGAA-3') and creAC (5'-GGACACTTGTACGG CCTG-3'). The characterization and stable of mutants were also according to Chi et al. [39].

\section{Acknowledgements \\ The authors are grateful to Dr. S. Anderson for English editing of the manuscript.}

\section{Authors' contributions \\ This project was conceived and designed by LLM, YL and ZPL. SC tested all the carotenoids profiles, constructed all the plasmids in this study. The genes expression profiles of astaxanthin synthesis pathway in different glucose concentration were carried out by MRW. All authors read and approved the final manuscript.}

\section{Funding}

This study was supported by the National Nature Science Foundation of China (grant No. 31400037). The funders had no role in the design of the study, collection, analysis, and interpretation of data, decision to publish, or preparation of the manuscript.

Availability of data and materials

All data and materials are available upon request.

\section{Ethics approval}

This article does not contain any studies with human participants or animals performed by any of the authors.

Consent for publication

Not applicable.

\section{Competing interests}

The authors declare that they have on competing interest.

\section{Author details}

${ }^{1}$ State Key Laboratory of Microbial Resources, Institute of Microbiology, Chinese Academy of Sciences, No. 1 West Beichen Road, Chaoyang District, Beijing 100101, People's Republic of China. ${ }^{2}$ State Key Laboratory of Agrobiotechnology and College of Biological Sciences, China Agricultural University, Beijing 100193, People's Republic of China.

Received: 2 January 2019 Accepted: 31 May 2019

Published online: 15 June 2019

\section{References}

1. Lu Y, Wang X, Feng J, Xie T, Si P, Wang W. Neuroprotective effect of astaxanthin on newborn rats exposed to prenatal maternal seizures. Brain Res Bull. 2019;148:63-9.

2. Afzal S, Garg S, Ishida Y, Terao K, Kaul SC, Wadhwa R. Rat Glioma cell-based functional characterization of anti-stress and protein Deaggregation activities in the marine carotenoids, Astaxanthin and Fucoxanthin. Mar Drugs. 2019;17(3).

3. Harada F, Morikawa T, Lennikov A, Mukwaya A, Schaupper M, Uehara O, et al. Protective effects of oral astaxanthin nanopowder against ultravioletinduced photokeratitis in mice. Oxidative Med Cell Longev. 2017;2017: 1956104-13.

4. Kobori M, Takahashi Y, Sakurai M, Ni Y, Chen G, Nagashimada M. Hepatic transcriptome profiles of mice with diet-induced nonalcoholic steatohepatitis treated with astaxanthin and vitamin E. Int J Med Sci. 2017; 18:593-607.

5. Komatsu T, Sasaki S, Manabe Y, Hirata T, Sugawara T. Preventive effect of dietary astaxanthin on UVA-induced skin photoaging in hairless mice. PLoS One. 2017;12:e0171178. 
6. Camera E, Mastrofrancesco A, Fabbri C, Daubrawa F, Picardo M, Sies H, et al. Astaxanthin, canthaxanthin and beta-carotene differently affect UVAinduced oxidative damage and expression of oxidative stress-responsive enzymes. Exp Dermatol. 2009;18:222-31.

7. Liu X, Shibata T, Hisaka S, Osawa T. Astaxanthin inhibits reactive oxygen species-mediated cellular toxicity in dopaminergic SH-SY5Y cells via mitochondria-targeted protective mechanism. Brain Res. 2009;1254:18-27.

8. Nishigaki I, Rajendran P, Venugopal R, Ekambaram G, Sakthisekaran D, Nishigaki Y. Cytoprotective role of astaxanthin against glycated protein/iron chelate-induced toxicity in human umbilical vein endothelial cells. Phytother Res. 2010;24:54-9.

9. Will OH 3rd, Sawtelle DE, Iverson P, Jorve K. Protection of Ustilago violacea from toluidine blue photosensitization and hydrogen peroxide induced killing and mitotic recombination by carotenes. Photochem Photobiol. 1988; 48:305-9.

10. Wolf AM, Asoh S, Hiranuma H, Ohsawa I, lio K, Satou A, et al. Astaxanthin protects mitochondrial redox state and functional integrity against oxidative stress. J Nutr Biochem. 2009;5:381-9.

11. Johnson EA. Phaffia rhodozyma: colorful odyssey. Int Microbiol. 2003;6:16974.

12. Johnson EA, Lewis MJ. Astaxanthin formation by the yeast Phaffia rhodozyma. Microbiology. 1979;115:173-83.

13. Schmidt I, Schewe H, Gassel S, Jin C, Buckingham J, Hümbelin M, et al. Biotechnological production of astaxanthin with Phaffia rhodozyam/ Xanthophyllomyces dendrorhous. Appl Microbiol Biotechnol. 2011;89:555-71.

14. Reynders MB, Rawlings DE, Harrison STL. Demonstration of the Crabtree effect in Phaffia rhodozyma during continuous and fed-batch cultivation. Biotechnol Lett. 1997;19:549-52.

15. Sandmann G. Carotenoid biosynthesis in microorganisms and plants. Eur J Biochem. 1994;223:7-24.

16. Alcaíno J, Bravo N, Córdova P, Marcoleta AE, Contreras G, Barahona S, et al. The involvement of Mig1 from Xanthophyllomyces dendrorhous in catabolic repression: an active mechanism contributing to the regulation of carotenoid production. PLoS One. 2016;11:9-33.

17. Córdova P, Alcaíno J, Bravo N, Barahona S, Sepúlveda D, Fernández-Lobato $\mathrm{M}$, et al. Regulation of carotenogenesis in the red yeast Xanthophyllomyces dendrorhous: the role of the transcriptional co-repressor complex Cyc8Tup1 involved in catabolic repression. Microb Cell Factories. 2016;15:193212.

18. Hara KY, Morita T, Mochizuki M, Yamamoto K, Ogino C, Araki M, et al. Development of a multi-gene expression system in Xanthophyllomyces dendrorhous. Microb Cell Factories. 2014;13:175-82.

19. Pan X, Wang B, Gerken HG, Lu Y, Ling X. Proteomic analysis of astaxanthin biosynthesis in Xanthophyllomyces dendrorhous in response to low carbon levels. Bioprocess Biosyst Eng. 2017:40:1091-100.

20. Yamamoto K, Hara KY, Morita T, Nishimura A, Sasaki D, Ishii J, et al. Enhancement of astaxanthin production in Xanthophyllomyces dendrorhous by efficient method for the complete deletion of genes. Microb Cell Factories. 2016;15:155-63.

21. Sieiro C, Poza M, de Miguel T, Villa TG. Genetic basis of microbial carotenogenesis. Int Microbiol. 2003;6:11-6.

22. Verdoes JC, Krubasik KP, Sandmann G, van Ooyen AJ. Isolation and functional characterisation of a novel type of carotenoid biosynthetic gene from Xanthophyllomyces dendrorhous. Mol Gen Genet. 1999;262:453-61.

23. Verdoes JC, Misawa N, van Ooyen AJ. Cloning and characterization of the astaxanthin. Biosynthetic gene encoding phytoene desaturase of Xanthophyllomyces dendrorhous. Biotechnol Bioeng. 1999;62:750-5.

24. Alvarez V, Rodriguez-Saiz M, de la Fuente JL, Gudina EJ, Godio RP, Martin JF, et al. The crtS gene of Xanthophyllomyces dendrorhous encodes a novel cytochrome-P450 hydroxylase involved in the conversion of beta-carotene into astaxanthin and other xanthophylls. Fungal Genet Biol. 2006;43:261-72.

25. Lodato P, Alcaino J, Barahona S, Niklitschek M, Carmona M, Wozniak A. Expression of the carotenoid biosynthesis genes in Xanthophyllomyces dendrorhous. Biol Res. 2007;40:73-84.

26. Marcoleta A, Niklitschek M, Wozniak A, Lozano C, Alcaino J, Baeza M. Glucose and ethanol-dependent transcriptional regulation of the astaxanthin biosynthesis pathway in Xanthophyllomyces dendrorhous. BMC Microbiol. 2011;11:190-201.

27. Miao L, Chi S, Tang Y, Su Z, Yin T, Guan G, et al. Astaxanthin biosynthesis is enhanced by high carotenogenic gene expression and decrease of fatty acids and ergosterol in a Phaffia rhodozyma mutant strain. FEMS Yeast Res. 2011;11:192-201.

28. Arrach N, Schmidhauser TJ, Avalos J. Mutants of the carotene cyclase domain of al-2 from Neurospora crassa. Mol Gen Genomics. 2002;266:91421.

29. Carattoli A, Romano N, Ballario P, Morelli G, Macino G. The Neurospora crassa carotenoid biosynthetic gene (albino 3) reveals highly conserved regions among prenyltransferases. J Biol Chem. 1991;266:5854-9.

30. Almeida ER, Cerda-Olmedo E. Gene expression in the regulation of carotene biosynthesis in Phycomyces. Curr Genet. 2008:53:129-37.

31. Arrach N, Fernandez-Martin R, Cerda-Olmedo E, Avalos J. A single gene for lycopene. cyclase, phytoene synthase, and regulation of carotene biosynthesis in Phycomyces. Proc Natl Acad Sci U S A. 2001;98:1687-92.

32. Schmidt AD, Heinekamp T, Matuschek M, Liebmann B, Bollschweiler C, Brakhage AA. Analysis of mating-dependent transcription of Blakeslea trispora carotenoid biosynthesis genes carB and carRA by quantitative realtime PCR. Appl Microbiol Biotechnol. 2005;67:549-55.

33. Lukacs G, Papp T, Somogyvari F, Csernetics A, Nyilasi I, Vagvolgyi C. Cloning of the Rhizomucor miehei 3-hydroxy-3-methylglutaryl-coenzyme a reductase gene and its heterologous expression in Mucor circinelloides. Antonie Van Leeuwenhoek. 2009;95:55-64.

34. Schimek C, Wostemeyer J. Carotene derivatives in sexual communication of zygomycete fungi. Phytochemistry. 2009;70:1867-75.

35. Estrada AF, Avalos J. The white collar protein WcoA of Fusarium fujikuroi is not essential for photocarotenogenesis, but is involved in the regulation of secondary metabolism and conidiation. Fungal Genet Biol. 2008:45:705-18.

36. Estrada AF, Avalos J. Regulation and targeted mutation of opsA, coding for the NOP-1 opsin orthologue in Fusarium fujikuroi. J Mol Biol. 2009;387:5973.

37. Miao L, Wang Y, Chi S, Yan J, Guan G, Hui B, et al. Reduction of fatty acid flux results in enhancement of astaxanthin synthesis in a mutant strain of Phaffia rhodozyma. J Ind Microbiol Biotechnol. 2010;37:595-602.

38. Montserrat V, Riera A, Fernández-Cid A, Herrero P, Moreno F. Hexokinase 2 is an intracellular glucose sensor of yeast cells that maintains the structure and activity of Mig1 repressor complex. J Biol Chem. 2016;291:7267-85.

39. Chi S, He Y, Ren J, Su Q, Liu X, Chen Z, et al. Overexpression of a bifunctional enzyme, CrtS, enhances astaxanthin synthesis through two pathways in Phaffia rhodozyma. Microb Cell Factories. 2015;14:90-102.

\section{Publisher's Note}

Springer Nature remains neutral with regard to jurisdictional claims in published maps and institutional affiliations.
Ready to submit your research? Choose BMC and benefit from:

- fast, convenient online submission

- thorough peer review by experienced researchers in your field

- rapid publication on acceptance

- support for research data, including large and complex data types

- gold Open Access which fosters wider collaboration and increased citations

- maximum visibility for your research: over $100 \mathrm{M}$ website views per year

At BMC, research is always in progress.

Learn more biomedcentral.com/submissions 